\title{
ON EQUISINGULAR FAMILIES OF ISOLATED SINGULARITIES
}

\author{
A. Nobile
}

Basic properties of a definition of equisingularity for families of (algebraic, analytic or algebroid) varieties, singular along a given section, are studied. The equisingularity condition is: given a family $p: X \rightarrow Y$, with a section $s$, it is required that the natural morphism $E \rightarrow Y$ be flat, where $E$ is the exceptional divisor of the blowing-up of $X$ with center the product of the ideal defining $s$ and the relative Jacobian ideal.

The following results hold: (a) This condition is invariant under base change (b) It implies equimultiplicity, the validity of the Whitney conditions and topological triviality along $s$ (c) If $Y$ is reduced, the condition holds over a dense open set of $Y$, whose complement is a subvariety of $Y$ (d) If $Y$ is smooth and the fibers of $p$ are plane curves, this definition agrees with Zariski's.

Introduction. In this article, we discuss some basic consequences of a possible definition of equisingularity, for families of isolated singularies of algebraic, algebroid and analytic varieties (cf. Definitions (1.1) and (1.4)). Our basic definition is closely related to one suggested by Hironaka years ago. In fact, around 1964, in his pioneering work on the Whitney conditions, he did the following: given a family of isolated singularies $\pi: X \rightarrow Y$ (say, to simplify, with $Y$ smooth), he took the blowing-up $Z$ of $X$ with center $I J$, where the ideal $I$ defines the singular locus of $X$, and $J$ is the Jacobian ideal of $\pi$, and after that the normalization $\widetilde{Z}$ of $Z$. If $\widetilde{E}$ is the subspace of $\widetilde{Z}$ corresponding to $I J Q_{\mathbb{Z}}$, he required that the composed morphism $\widetilde{h}: \widetilde{E} \rightarrow Y$ be flat (cf. [5]). Since then, some interesting topological results were obtained with these techniques (cf. [9]); however, apparently no careful study of a theory of equisingularity based on these ideas has been attempted. Here we study some basic results related to the definition that is obtained when, in Hironaka's process, the normalization is omitted. We call this "condition $\mathscr{E}$ ". This seems a reasonable requirement, if we are interested in having good functorial properties and in accepting nonreduced spaces (e.g., infinitesimal deformations). More precisely, in $\$ 1$ we present the basic definitions, and we prove that this theory has some of the fundamental "nice" properties that a good theory of equisingularity should have (equimultiplicity, topological triviality, openness, etc). The proofs of $\S 1$ are simple applications of known 
theorems. In $\S 2$, we check that this notion "behaves well" under base change. In $\S 3$ we present the less trivial result of this paper, namely that for families of plane curves (with smooth parameter space), our definition coincides with Zariski's (i.e., with the requirement that Whitney's conditions hold). A certain family of surfaces, introduced by Briançon and Speder in [1], can be used to give an example which shows that condition $\mathscr{E}$ is, in higher dimension, strictly stronger than Whitney's conditions. There is a $\S 0$, where the basic terminology and notation is explained.

It should be noted that the functorial properties shown in $\S 2$ hold without the requirement that the parameter space be smooth or reduced, hence the basic definitions apply to nonreduced spaces; in particular one can introduce a theory of deformations of a singularity, satisfying condition $\mathscr{E}$. More precisely, given an algebroid isolated singularity $X_{0}=\operatorname{Spec}\left(B_{0}\right), B_{0}=k\left[\left[x_{1}, \cdots, x_{n}\right]\right] / G$, with $G$ an ideal, $k$ a field as in $(0.1)$, then the correspondence $A \rightarrow E(A)$, where $E(A)$ is the set of isomorphism classes of families $\mathscr{F}=(\pi, X, Y, s)$, (cf. (1.2), here $Y=\operatorname{Spec}(A), A$ artinian in $\mathscr{C}$ (cf. (0.4)) and $\pi: X \rightarrow Y$ a deformation of $X_{0}$ ), which satisfy condition $\mathscr{C}$, naturally extends to a functor $\mathscr{C} \rightarrow$ (Sets). Here, by "an isomorphism", we mean an isomorphism that respects the section.

It can be proved that this functor always admits a hull, or versal deformation. This result, as well as other finer results on infinitesimal equisingular deformatiors (in the sense of condition $\mathscr{E}$ ), will be presented in another paper.

o. Notations and terminology.

(0.1) In this paper $k$ denotes an algebraically closed field of zero characteristic. All rings are commutative, with identity, and ring homomorphisms respect the identities.

$(0.2)$ The word: "scheme" means algebraic scheme over $k$.

(0.3) The category of complex analytic spaces will be denoted by $A n$.

(0.4) The symbol $\mathscr{C}$ will denote the category of $k$-algebras which are of the form $k\left[\left[X_{1}, \cdots, X_{n}\right]\right] / G$, with $G$ an ideal, with the usual $k$-algebra homomorphisms (which are necessarily local).

(0.5) Sometimes, to simplify the notation, a sheaf of ideals in a sheaf of rings is simply called an ideal. If $X$ is a scheme, or an analytic space, and $\mathscr{T}$ is a sheaf of ideals in $\mathscr{O}_{X}$, the symbol $\mathscr{B}(X, \mathscr{T})$ denotes the blowing-up of $X$ with center $\mathscr{T}$.

1. Basic definitions and results.

(1.1) In this paper we shall deal with families of singularities, in the algebraic, complex analytic and formal cases. In the algebraic 
case, the basic definition that we adopt is the following one (cf. [5]).

DEFINITION 1.2. A family of singularities is a system $\mathscr{F}=$ $(p, X, Y, s)$, where $p: X \rightarrow Y$ is a flat morphism of schemes, $s$ is a section, and all the fibers $X_{y}=p^{-1}(y), y \in Y$, are reduced and equidimensional.

If, moreover, $X_{y}$ is smooth off $s(Y)$ we say that $\mathscr{F}$ is a family of isolated singularies.

(1.3) There are obvious analogous definitions working in the categories $A n$ and $\mathscr{C}$. Working with analytic spaces there is also a notion of a germ of a family of singularities, substituting in $1.2 X$ and $Y$ by germs of spaces, etc. Given an analytic family of singularities in the sense of 1.2 , and a point $y \in Y$, there is an induced germ of family of singularities at $y$, which will be denoted by $\mathscr{F}_{y}$. Given $\mathscr{F}$ as in 1.2 and a morphism $Y^{\prime} \rightarrow Y$, the pullback of $\mathscr{F}$ over $Y^{\prime}$ is defined in an obvious way.

(1.3) We recall the definition of the Jacobian ideal of a family. Again we discuss the algebraic case, the other two being similar. Let $\mathscr{F}$ be a family of singularities (notations as in (1.2)). Locally over $Y$, we may assume that $p$ is induced by the inclusion of rings $A \rightarrow B=A\left[X_{0}, \cdots, X_{n}\right] / L, L=\left(f_{1}, \cdots, f_{m}\right)$ an ideal of $A\left[X_{0}, \cdots, X_{n}\right]$. Let $r=\operatorname{dim} X_{y}(y$ any closed point of $Y)$, and $J$ the ideal of $B$ generated by the $(n-r) \times(n-r)$ minors of the Jacobian matrix $\left(\partial f_{i} / \partial x_{j}\right), i=1, \cdots, m, j=1, \cdots, n$. This ideal is independent of the representation $B=A[X] / L$, and, by taking a covering of $Y$ by affine open sets $\operatorname{Spec}(A)$ as above, we get a sheaf of ideals $\mathscr{J}$ of $\mathcal{O}_{x}$, called the sheaf of Jacobian ideals of $\mathscr{F}$.

(1.4) The $H$-ideal of $\mathscr{F}$, denoted by $H(\mathscr{F})$ is the product $\mathscr{J} \mathcal{J}$, where $\mathscr{T}$ is the ideal defining $s(Y)$ and $\mathscr{J}$ the Jacobian ideal of $\mathscr{F}$. Given the family $\mathscr{F}$, the $H$-transform $Z$ of $X$ (or, more precisely, of $\mathscr{F}$ ) is the monoidal transform of $X$ with center $H(\mathscr{F})$, its exceptional divisor is called the $H$-divisor of $\mathscr{F}$. We have morphisms:

$$
E \longrightarrow Z \longrightarrow X \longrightarrow Y
$$

Let $h: E \rightarrow Y$ be the composition. We say that the family of isolated singularities $\mathscr{F}$ satisfies "condition $\mathscr{E}$ ", or that it is equisingular, if $h$ is flat (cf. [5], pg 9).

(1.5) In this paragraph we work in the analytic category. We assume $Y$ smooth. It is well known that the flatness of $h$ implies: all the fibers of $E_{\text {red }} \rightarrow Y$ have the same dimension. In [9] it is proved that this equidimensionality condition implies: the pair 
$(X-s(Y), Y)$ satisfies the Whitney conditions $A$ and $B$. By the results of [6], it follows that $X$ is equimultiple along $Y$.

(1.6) Another nice property of "condition $\mathscr{E}$ " is the following one: given a family of analytic spaces $\mathscr{F}$ (notations as in 1.2), then $U=\{y \in Y /$ condition $\mathscr{E}$ holds at $y\}$ is the complement of a closed subspace $W$ of $Y$. If $Y$ is reduced, then $U$ is dense in $Y$. In fact, let $E$ be the $H$-divisor of $\mathscr{F}, h: E \rightarrow Y$ the surjective morphism of (1.4.1), and $G=\{z \in E / h$ is flat at $x\}$ (note that $E-G$ is a closed analytic set). Then, condition $\mathscr{E}$ does not hold at $y$ if and only if $y \in h(E-G)=W$. Since $h$ is proper, $W$ is a closed analytic set. If $Y$ is reduced, the surjective morphism $h$ will be flat over an open dense set of $Y$, hence $Y-W$ will be dense.

\section{Functorial properties.}

(2.1) In the paragraphs 2.2 and 2.3 we review some basic facts on monoidal transforms, which we could not find in the literature. Our goal is to prove Theorem 2.4, which says that equisingular families in the sense of condition $\mathscr{E}$ "behave well" with respect to base change.

Lemma 2.2. Let $p: X^{\prime} \rightarrow X$ be a morphism of schemes, $I$ a sheaf of ideals of $C_{X}^{\prime}, I^{\prime}=I O_{X^{\prime}}, Z=\mathscr{B}(X, I), E$ the exceptional divisor; assume $g^{-1}(E)$ (where $g: X^{\prime} \times Z \rightarrow Z$ is the projection) is a divisor in $X^{\prime} \times{ }_{X} Z$. Then, there is a canonical isomorphism $X^{\prime} \times Z \cong$ $Z^{\prime}=\mathscr{P}\left(X^{\prime}, I^{\prime}\right)$, and $g^{-1}(E)$ corresponds to the exceptional divisor of $Z^{\prime}$.

Proof. The surjection $\left(\oplus I^{n}\right) \bigotimes_{O_{X}} O_{X^{\prime}}^{\prime} \rightarrow \bigoplus I^{n} O_{X^{\prime}}$ induces a closed immersion $q: Z^{\prime} \rightarrow X^{\prime} \times_{X} Z$, which makes the (solid) diagram:

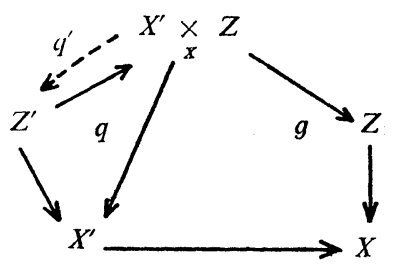

commutative. Since $g^{-1}(E)$ is a divisor, the universal property of the monoidal transform implies the existence of the dotted arrow $q^{\prime}$. It is clear that $q$ and $q^{\prime}$ are inverse to each other, and that $g^{-1}(E)$ becomes the exceptional divisor of $Z^{\prime}$.

Remark 2.3. If $f: X \rightarrow Y$ is a morphism of schemes, $D \subset X$ is an effective divisor, $X-D-Y$ (induced by $f$ ) and the composition 
$D \subset X \rightarrow Y$ are flat, then $f$ is flat, and $D$ is a relative Cartier divisor (cf. [8]).

THEOREM 2.4. Let $\mathscr{F}=(p, X, Y, s)$ be an equisingular family, $Y^{\prime} \rightarrow Y$ a morphism of schemes, $\mathscr{F}^{\prime}$ the pull-back of $\mathscr{F}$ to $Y^{\prime}$. Then, $\mathscr{F}^{\prime}$ is equisingular.

Proof. Let $\mathscr{Y}=(p, X, Y, s), \pi: Z \rightarrow X$ the $H$-transform of $X$, $E$ its exceptional divisor. By 2.3 and $1.4, E$ is a relative divisor of $Z$ over $Y$, then (cf. [4], pg 332), its pull-back $E^{\prime}$ to $Y^{\prime} \times X$ is a divisor. By 2.2 , the diagram

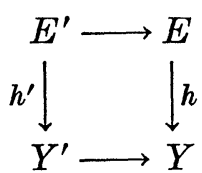

is cartesian. Since $h$ is flat, $h^{\prime}$ is flat, i.e., $\mathscr{F}^{\prime}$ is equisingular.

REMARK 2.5. In the definition of equisingularity (1.4), we are dealing with a family of isolated singularities, in the sense of (1.1). However, the "isolated singularities" condition is implied by the other requirements of (1.4). Precisely, let $\mathscr{F}=(p, X, Y, s)$ be a family of singularities with $Y$ connected, $y_{0} \in Y$ a closed point, assume that $X_{0}=p^{-1}\left(y_{0}\right)$ has a single singular point, at $s\left(y_{0}\right)$ and (with notations as in (1.4)) that $h$ is flat. Then, for each $y \in Y, X_{Y}$ has an isolated singularity at $s(y)$.

To see this, note that in the proof of 2.4 we did not use the fact that the fibers have isolated singularities, hence the same argument shows that the property: " $h$ flat" is stable under base change. So, we may assume $Y$ to be integral. Clearly, it suffices to show: let $S=\operatorname{Spec}\left(C_{Y, y_{0}}\right), \mathscr{F}_{1}=\left(p, X_{1}, S, s_{1}\right)$ the pull-back of $\mathscr{F}$ over $S, t$ the general point of $S$, then $p_{1}^{-1}(t)$ has a singularity at $s_{1}(t)$ (here we assume $X_{0}$ is actually singular at $s\left(y_{0}\right)$, otherwise the statement is trivial). The $H$-ideal of $\mathscr{F}_{1}$ looks, near $y_{0} \in S$, as $I J$ (with $I$ defining $s_{1}(S), J$ the relative Jacobian). At $y_{0}, I$ and $J$ are nonunits. On the $H$-transform $Z_{1}$, they become invertible, in particular $J$ defines a divisor $D$. If we assume, by contradiction, that $p_{1}^{-1}(t)$ is nonsingular, then the stalk of $J$ at $s_{1}(t)$ is the unit ideal. But then $D_{\text {red }}$ (a union of irreducible components of the $H$-divisor $E_{1}$ ) maps into a proper subscheme of $S$, an impossibility since $E_{1} \rightarrow S$ is flat.

(2.6) Of course there are results analogous to those presented in this section for the categories $A n$ and $\mathscr{C}$.

3. The case of plane curves. In this section we prove that 
for families of plane curves (with smooth parameter), Definition 1.4 agrees with Zariski's definition of equisingularity. For simplicity, we shall work with complex analytic spaces.

THEOREM 3.1. Let $\mathscr{F}=(p, X, Y, s)$ be an analytic family of singularities, where $X_{y}$ is a plane curve, for all $y \in Y$, and $Y$ is smooth. Then, $\mathscr{F}$ satisfies condition $\mathscr{E}$ if and only if it is Zariski equisingular.

Proof. As remarked in 1.5, condition $\mathscr{E}$ implies the validity of Whitney conditions along $s(Y)$, which is equivalent to Zariski equisingularity (cf. [11] Theorem 8.1).

To see the converse, we need some auxiliary results. Several times we shall use germs (of spaces and families); to simplify the notation we shall sometimes write just $X$ (resp. $\mathscr{F}$ ) to denote the germ $(X, x)$ (resp. the germ of a family $\mathscr{F}_{y}$ ).

Recall that given a germ of a plane curve $(C, 0)$, there is a versal deformation $g:(X, 0) \rightarrow(U, 0)$ (cf. [10], Chapter III, in that paper what we call "versal" is called mini-versal). The smooth space $U$ contains a smooth subspace $D_{\mu}=\{u \in U / x$ is in the discriminant $D$ of $g$, and $m(u)=m(0)\}(m=$ multiplicity of a point of $D)$. If $\ell: X_{\mu} \rightarrow D_{\mu}$ is the pull-back of $g$, then $\ell$ admits natural section $\sigma$ (to define it, note that the fibers $g^{-1}(u), u \in D_{\mu}$ have a unique singularity, $s_{u}$, with same Milnor number as $\left(g^{-1}(0), 0\right), \sigma$ is defined by $\left.\sigma(u)=s_{u}\right)$, and $\mathscr{G}=\left(\ell, X_{\mu}, D_{\mu}, \sigma\right)$ is a (Zariski) equisingular versal deformation of $C$. In the paragraphs (3.8) to (3.16) we shall prove the following.

Proposition 3.2. There is a reduced subspace $F$ of $D_{\mu}$, such that the pull-back $\mathscr{G}_{1}=(q, G, F, s)$ of $\mathscr{G}$ to $F$ has the following property: $\mathscr{C}$ satisfies condition $\mathscr{E}$ and if $\left(X^{\prime}, x\right) \rightarrow(S, s)$ is a deformation of $(C, 0)$, with reduced space $S$, then it satisfies condition $\mathscr{E}$ if and only if morphism $(S, s) \rightarrow(U, 0)$ (obtained by versality of g) factors through $F$.

We shall also need:

Proposition 3.3. If $\mathscr{F}=(p, X, Y, s)$ is a deformation of $a$ germ of a plane curve $C$, with $Y$ smooth, one-dimensional, then $\mathscr{F}$ is Zariski equisingular if and only if it satisfies condition $\mathscr{E}$.

(3.4) We prove 3.3 in (3.7). With 3.2 and 3.3, Theorem 3.1 easily follows, with the following well-known argument (see [10], pg 358, Proof of 2.9). To show that "Zariski equisingularity 
implies condition $\mathscr{E} "$, clearly it suffices to show: for any germ of plane curve $(C, 0)$, we have $D_{\mu}=F$ (notations of (3.2)). Were $F \varsubsetneqq D_{\mu}$, then there would be a curve $\Lambda \subset D_{\mu}$, such that $\Lambda \cap F=\{0\}$. The pull-back of $X_{\mu} \rightarrow D_{\mu}$ to $\Lambda^{\prime}=$ normalization of $\Lambda$, is Zariski equisingular, hence by (3.3) it satisfies condition $\mathscr{E}$. But the resulting morphism $\Lambda^{\prime} \rightarrow U$ does not factor through $F$, a contradiction to (3.2).

Before proving the propositions, we need:

Lemma 3.5. Let $\mathscr{F}$ be a family of plane curves as in (3.1), which is Zariski equisingular, let $Z \rightarrow X$ be the H-transform of $X$, $f$ the composition $Z \rightarrow X \stackrel{p}{\rightarrow} Y$, and $b \in Y$. Then, $f^{-1}(b)$ is isomorphic to the H-transform of $X_{b}$, parametrized by a point, with the section defined by $s(b)$ (and hence $f^{-1}(b)$ is reduced).

Proof. Clearly we may assume that, near $s(b), X \subset C^{n+2}$, defined by $g=0, g \in C\left\{u_{1}, \cdots, u_{n}, x, y\right\}, Y \subset X$, defined by $x=y=0$, moreover neither the $x$-axis nor the $y$-axis is tangent to $p^{-1}(b): f(0, x, y)=0$ at the origin $0=s(b)$. Zariski equisingularity implies: the irreducible components $X_{1}, \cdots, X_{d}$ of $X$ (near 0 ) admit parametrizations:

$$
x=t^{n_{i}}, \quad y=\sum_{j=n}^{\infty} a_{j}^{(i)}(u) t^{j}, \quad u_{k}=u_{k}, \quad k=1, \cdots, n,
$$

here, $i=1, \cdots, d, u=\left(u_{1}, \cdots, u_{n}\right)$ and $a_{j}^{(i)}(0) \neq 0$. It is readily verified that (for a small neighborhood $S \Subset Y$ of 0 ) then open $f^{-1}(S)$ of the $H$-transform of $X$ is contained in $C^{n+4}$ (of coordinates $\left(v_{1}, \cdots, v_{n}\right.$, $\left.w_{1}, \cdots, w_{4}\right)$, and it has $d$ components which admit parametrizations:

$$
\begin{aligned}
v_{k} & =u_{k}, \quad k=1, \cdots, n \\
w_{1} & =t^{n}, \quad w_{2}=\sum_{j=n_{i}}^{\infty} a_{j}^{i}(u) t^{j} \\
w_{3} & =a_{n_{i}}(u)+\frac{n+1}{n} a_{n_{i}+1}(u) t+\cdots \\
w_{4} & =\left(a_{n_{i}}(u)+a_{n_{i}+1}(u)+\cdots\right)^{-1}\left(\text { recall } a_{n_{i}}(0) \neq 0\right) .
\end{aligned}
$$

(In fact, the "relevant" open of the $H$-transform is the analytic spectrum of $\mathcal{O}_{s}\left[f_{x} / f_{y}, x / y\right]$.) Now it becomes clear that $f^{-1}(b)$ is the curve with $d$ components parametrized by the series obtained from (3.5.2) by making $u_{i}=0, i=1, \cdots, n$, which (as before) is the $H$ transform of $X_{b}$, with the section defined by the point $s(b)$.

(3.7) Proof of Proposition 3.3. Assume $\mathscr{F}$ is Zariski equisingular. Consider the $H$-transform $Z \stackrel{g}{\rightarrow} X$, let $E$ be the exceptional divisor, and $f=p g$. Since $Z$ is reduced (hence it has no embedded points) and no component of $Z$ maps into a point of the smooth 
curve $Y, f$ is flat. Since (by Lemma 3.5) the fibers of $f$ are reduced curves, and hence Cohen-Macaulay, by the flatness of $f$ the space $Z$ is Cohen-Macaulay. Hence, the divisor $E \subset Z$ is a CohenMacaulay space, and the flatness of $E$ over $Y$ is equivalent to the equidimensionality of the fibers. But this is insured by [2], Section III, since $\mathscr{F}$, being Zariski equisingular, satisfies the Whitney conditions. The other implication always holds, so Proposition 3.3 is proved.

(3.8) We begin the proof of Proposition 3.2. Consider (using the notations introduced before 3.2 ) the $H$-transform $Z \rightarrow X_{1}$ of the family $\mathscr{G}$, let $E$ be the $H$-divisor. A priori, we do not know whether $E$ is flat over $X_{\prime \prime}$ or not. By the theory of flattening stratifications (see [3], pg. 156) there is a sub-germ $F^{\prime}$ of $D_{\mu}$ such that $E^{\prime}=E \times_{D_{\mu^{\prime \prime}}} F^{\prime}$ is flat over $F^{\prime}$, and any morphism of germs $v: T \rightarrow D_{\mu}$ such that $E \times_{D_{\mu}} T$ is flat, factors through $F^{\prime}$. Let $F=F_{\text {red }}^{\prime}$, then $F$ has a similar universal property, but with respect to morphisms $v: T \rightarrow D_{u}$ with $T$ reduced. Consider the $H$-transform $Z_{1} \rightarrow F$ of $\mathscr{C}_{1}=(q, G, F, s)=\mathscr{C} \times_{D_{1 \prime}} F$, with $H$-divisor $E_{1}$. We have the following result, to be proved in (3.13).

LEMмA 3.9. In the following diagram, the squares are cartesian:

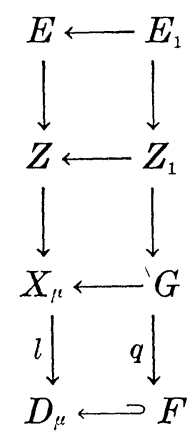

(3.10) By this lemma, $\mathscr{C}_{1}$ satisfies condition $\mathscr{E}$. Assume now that a deformation of $(C, 0)$, say $\mathscr{O}_{2}=\left(p^{\prime}, X^{\prime}, S, s^{\prime}\right)$ (with $S$ reduced and $\left(p^{-1}\left(t_{0}\right), s^{\prime}\left(t_{0}\right) \approx(C, 0)\right)$, satisfies condition $\mathscr{E}$. Since all germs $\left(X_{t}, s^{\prime}(t)\right), t \in S$ have the same Milnor number, and since $S$ is reduced, any morphism $S \rightarrow U$ (associated to $X^{\prime} \rightarrow S$ by versality of $X \rightarrow U$ ) factors through $D_{\mu}$. We claim:

LEMMA 3.11. There is a canonical isomorphism:

$$
E \times_{D_{\mu}} S \approx E_{2}
$$

where $E_{2}$ is the H-divisor of $\mathscr{B}_{2}$. 
(3.12) This lemma is proved in (3.16). By (3.11), the morphism $S \rightarrow U$ factors through $F$. Conversely, if $S \rightarrow U$ factors through $F$, by Theorem $2.4 p^{\prime}: X^{\prime} \rightarrow S$ (or, more precisely, $\left(p^{\prime}, X^{\prime}, S, s^{\prime}\right.$ ), where $s^{\prime}$ is induced by the section of $\mathscr{G}_{1}$ ) satisfies condition $\mathscr{E}$.

(3.13) To complete the proof of Proposition 3.2, we show Lemmas 3.9 and 3.11. To prove 3.9 , note that we have a commutative diagram (cf. the proof of (2.2))

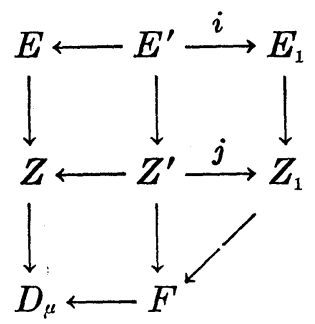

here, $E^{\prime}=E \times_{D_{\mu}} F \quad Z^{\prime}=Z \times_{D_{\mu}} F$, and $i, j$ are closed immersions. By using Lemma 3.5, it is easy to see that actually $i$ and $j$ are homeomorphisms. If we prove that $i$ is an isomorphism, it follows that $j$ is an isomorphism, concluding the proof. In fact, if $i$ is an isomorphism then $E_{1}$ is flat over $F$ (since $E^{\prime}$ is flat, by assumption). Then, by Remark 2.3, $Z_{1}$ is flat over $F$. Since, by (3.5), $i$ induces an isomorphism of the fibers of $E^{\prime} \rightarrow F$ and $E_{1} \rightarrow F$, the following well-known result (cf. [7], 20E) immediately implies that $j$ is an isomorphism.

LEMMA 3.14. Let $A \rightarrow B$ be a local homomorphism of local noetherian rings, $M \stackrel{u}{\rightarrow} N$ an epimorphism of finite B-modules, with $N$ flat over $A$. Assume $M \bigotimes_{A} k \rightarrow N \bigotimes_{A} k$ (with $k$ the residue field of $A$ ) is injective. Then, $u$ is an isomorphism.

So, to conclude the proof we must check that $i$ is an isomorphism. In veiw of Lemma 3.5, this is an immediate consequence of the following algebraic lemma.

LEMma 3.15. Let $h: B \rightarrow B^{\prime}$ be a surjective homomorphism of $A$-algebras, where $A$ is a noetherian reduced ring. Assume that $B$ is A-flat, and that for any minimal prime ideal $P$ of $A$, the induced map: $h(P): B \bigotimes_{A} k(P) \rightarrow B^{\prime} \bigotimes_{A} k(P)$ (where $k(P)$ is the field of fractions of $A / P)$ is an isomorphism. Then, $h$ is an isomorphism.

Proof. It suffices to show that $h$ is injective. First, note that for any minimal prime ideal $P$, the induced $\operatorname{map} h \otimes 1: B \otimes_{A} A / P \rightarrow$ $B^{\prime} \otimes_{A} A / P$ is an isomorphism. Clearly it is onto, to see the injectivity consider the commutative diagram: 


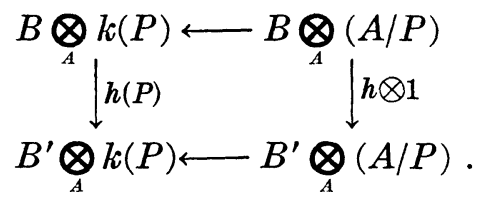

Since $B$ is $A$-flat, $\alpha$ is injective, hence $h \otimes 1$ is injective. Now, let $P_{1}, \cdots, P_{r}$ be the minimal primes of $A$. Consider the commutative diagram:

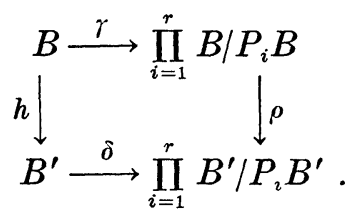

We just saw that $\rho$ is injective, and $\gamma$ can be identified to the homomorphism:

$$
1 \otimes j: B \otimes A \longrightarrow B \otimes \prod_{i=1}^{r}\left(A / P_{i}\right),
$$

where $j: A \rightarrow \prod_{i=1}^{r}\left(A / P_{i}\right)$ is the canonical injection (recall that $A$ is reduced). By the $A$-flatness of $B, \gamma$ is injective, hence $\delta h=\rho \gamma$ is injective, hence $h$ is injective. This proves Lemma 3.15.

(3.16) Proof of Lemma 3.11. Let $Z$, be the $H$-transform of $g_{2}$. Then there is a closed immersion $j: Z_{2} \rightarrow Z \times_{D_{\mu}} S$ (cf. (2.2)). Using Lemma 3.5 we see that $j$ induces an isomorphism of the fibers over the center $s$ of the germ $S$ (both fibers are naturally isomorphic to the $M$-transform of $(C, 0)$ ). Since $Z_{2}$ is flat over $S$, Lemma 3.14 implies that $j$ is an isomorphism. Now it is clear that we get the isomorphism of exceptional divisors of (3.11).

This completes the proof of Proposition 3.2.

(3.17) A natural question is the following: is "condition $\mathscr{E}$ " equivalent to "Whitney conditions", when we deal with a family of singularities of dimension $>1$ The answer is "no", as the following example shows.

(3.18) ExAmple. Consider the family of singular surfaces (parametrized by $t$, with trivial section):

$$
f=z^{3}+t x^{4} z+x^{6}+y^{6}
$$

(cf. [1]). In [1] it is shown that this family satisfies Whitney conditions along the $t$-axis $T$. But consider now its $H$-transform (to simplify, we work algebraically, i.e., with $\operatorname{Spec}(A), A=C[x, y, z, t] /$ $(f))$. Then, an affine of the $H$-divisor is given by the spectrum of 


$$
B=C\left[y / x, z / x, f_{x} / f_{y}, f_{2} / f_{y}\right] /\left(f, y^{5} x\right)
$$

which can be written as:

$$
B=k\left[t, x, y / x, z / x,(t z) / y^{2}, \quad\left(t x^{4}+3 z^{2}\right) / y^{5}\right] /\left(f, y^{5} x\right) .
$$

The $k[t]$-algebra $B$ is not flat: in fact, $t\left(x y^{3} z\right)=\left(t z / y^{2}\right) \cdot\left(y^{5} x\right)=0$ in $B$, however, it is not difficult to chech that $x y^{3} z \neq 0$ in $B$. Thus, $t$ becomes a zero divisor in $B$, and $B$ is not $A$-flat. Hence, this family does not satisfy condition $\mathscr{E}$.

\section{REFERENCES}

1. J. Briançon and J. P. Speder, Familles équisinguliéres de surfaces á singularité isolee, C. R. Acad. Sci. Paris, t. 280, Ser. A., (1975), 1013-1016.

2. - Les Conditions de Whitney impliquent $\mu^{(*)}$ constant, Preprint, U. de Nice, 1975.

3. G. Fischer, Complex Analytic Geometry, Lectures Notes in Math., No. 538, Springer.

4. A. Grothendieck, Éléments de Géométrie Algébrique, IV, 4e Partie, Publ. Math. I.M.E.S. No. 32, 1967.

5. H. Hironaka, Equivalence and Deformations of Isolated Singularities, Notes of the Woods Hole Conference on Alg. Geometry, 1964.

6. - Normal Cones in Analytic Whitney Stratifications, Publ. Math. I.H.E.S. No. 36, (1969), 127-138.

7. H. Matsumura, Commutative Algebra, W. A. Benjamin, N.Y., 1970.

8. A. Nobile, A note on flat algebras, Proc. Amer. Math. Soc., 64 (1977), 206-208.

9. J. P. Speder, Éclatements Jacobiens et Conditions de Whitney, Asterisque 7 et 8 , (1973), 47-66.

10. B. Teissier, Cycles Evanescentes, Sections Planes et Conditions de Whitney, Asterisque 7 and 8, (1973), 285-362.

11. O. Zariski, Studies on Equisingularity II, Amer. J. Math., 87 (1965), 972-1006.

Received July 15, 1977.

LOUISIANA STATE UNIVERSITY

BATON ROUGE, LA 70803 
